\title{
Self-Dual Space-Times with Cosmological Constant
}

\author{
R. S. Ward \\ School of Mathematics, Trinity College, Dublin, Ireland
}

\begin{abstract}
It is shown that self-dual solutions of Einstein's equations, with cosmological constant $\lambda$, correspond to certain complex manifolds. This result generalizes the work of Penrose [1], who dealt with the case $\lambda=0$.
\end{abstract}

\section{Introduction}

Recently there has been much interest in four-dimensional spaces which have self-dual conformal curvature, and which satisfy Einstein's vacuum equations (with or without cosmological constant) [1-5]. This is despite the fact that such spaces cannot have Lorentzian signature, and therefore have no direct application to classical gravity. The work in the papers listed above seems to suggest that self-dual solutions underlie gravity; that they are (in some sense) its basic constituents. Be that as it may, there is no doubt that self-dual solutions are a good deal easier to characterize and construct then more general solutions. This paper presents a characterization of self-dual solutions in terms of complex manifolds; it is essentially a generalization of Penrose's 'Nonlinear Gravitons and Curved Twistor Theory' [1], which deals only with the case of zero cosmological constant. Penrose's result has proved to be very useful for constructing and understanding self-dual solutions of Einstein's equations $[6,7]$. In addition, solutions of the wave equation $\left(\square+\frac{1}{6} R\right) \phi=0$ and of massless field equations of higher spin, in a self-dual gravitational background, may be neatly characterized in terms of certain sheaf cohomology groups [8]. Using this, one can explicitly construct Green's functions for these equations in a very natural way [9].

The remainder of this section is devoted to setting up some notation and conventions. Then in Sect. 2 the main theorem is presented, establishing a one-toone correspondence between self-dual solutions of Einstein's equations and deformations of flat twistor space which preserve certain differential forms. Some details of the construction and proof are relegated to Sect. 3 and an Appendix. In Sect. 4 the $C P_{2}$ gravitational instanton $[10,11]$ is discussed by way of example.

The following index conventions are used: $a, b, \ldots$ are 4-dimensional spacetime indices, $A, B, \ldots, A^{\prime}, B^{\prime}, \ldots$ are 2 -dimensional spinor indices, and $\alpha, \beta, \ldots$ are 4-dimensional twistor indices. The tangent space $T$ at a point of space-time is isomorphic to the tensor product of the unprimed spin-space $S$ and the primed 
spin-space $S^{\prime}[12]$ :

$$
T \cong S \otimes S^{\prime}
$$

This isomorphism is expressed via the Infeld-van der Waerden symbols $\sigma^{a}{ }_{A A^{\prime}}$ and $\sigma_{a}{ }^{A A^{\prime}}$; in other words, a vector $v^{a}$ corresponds to a spinor $v^{A A^{\prime}}$ according to

$$
\begin{aligned}
v^{a} & =\sigma_{A A^{\prime}}^{a} v^{A A^{\prime}}, \\
v^{A A^{\prime}} & =\sigma_{a}{ }^{A A^{\prime}} v^{a} .
\end{aligned}
$$

Occasionally the equations (1.2) are abbreviated to $v^{a}=v^{A A^{\prime}}$; this is in accordance with the abstract index notation [12]. Symmetrization (or skew-symmetrization) is indicated by round (or square) brackets enclosing the relevant indices.

The spin-spaces $S$ and $S^{\prime}$ are equipped with complex 2-forms $e_{A B}$ and $e_{A^{\prime} B^{\prime}}$ respectively. The tensor product of these forms corresponds to the space-time metric:

$$
g_{a b}=e_{A B} e_{A^{\prime} B^{\prime}} .
$$

The important point to remember is that these forms determine the metric: later on we shall define a metric by specifying $e_{A B}$ and $e_{A^{\prime} B^{\prime}}$.

Let $M$ be a 4-dimensional complex manifold with a holomorphic (complexRiemannian) metric $g_{a b}$; we shall call such a space a complex space-time. The Weyl conformal curvature tensor $C_{a b c d}$ and the Ricci tensor $R_{a b}$ of $M$ have the familiar decompositions $[12,1]$

$$
\begin{aligned}
C_{a b c d} & =\Psi_{A B C D} e_{A^{\prime} B^{\prime}} e_{C^{\prime} D^{\prime}}+\widetilde{\Psi}_{A^{\prime} B^{\prime} C^{\prime} D^{\prime}} e_{A B} e_{C D}, \\
R_{a b} & =-2 \Phi_{a b}+6 \Lambda g_{a b} .
\end{aligned}
$$

Here $\Psi_{A B C D}$ and $\widetilde{\Psi}_{A^{\prime} B^{\prime} C^{\prime} D^{\prime}}$ correspond, respectively, to the anti-self-dual and self-dual parts of $C_{a b c d} ;-2 \Phi_{a b}$ is the trace-free part of $R_{a b}$; and $24 \Lambda$ is the scalar curvature $R=R_{a}{ }^{a}$. (The sign conventions for the curvature tensor are $2 \nabla_{[a} \nabla_{b]} v^{d}=$ $R_{a b c}{ }^{d} v^{c}, R_{a b}=R_{a c b}^{c}$.) The spinor Ricci identities are [12]

$$
\begin{aligned}
& \square_{A^{\prime} B^{\prime}} \xi_{A}=\Phi_{A B A^{\prime} B^{\prime}} \xi^{B}, \\
& \square_{A B} \xi_{C}=\Psi_{A B C D} \xi^{D}-2 \Lambda \xi_{(A} e_{B) C}, \\
& \square_{A B} \xi_{A^{\prime}}=\Phi_{A B A^{\prime} B^{\prime}} \xi^{B^{\prime}} \\
& \square_{A^{\prime} B^{\prime}} \xi_{C^{\prime}}=\widetilde{\Psi}_{A^{\prime} B^{\prime} C^{\prime} D^{\prime}} \xi^{D^{\prime}}-2 \Lambda \xi_{\left(A^{\prime}\right.}, e_{\left.B^{\prime}\right) C^{\prime}}
\end{aligned}
$$

where $\square_{A^{\prime} B^{\prime}}=\nabla_{A\left(A^{\prime}\right.} \nabla_{\left.B^{\prime}\right)}{ }^{A}$ and $\square_{A B}=\nabla_{A^{\prime}\left(A^{\prime}\right.} \nabla_{B)}{ }^{A^{\prime}}$ are, respectively, the self-dual and anti-self-dual parts of the commutator $\nabla_{a} \nabla_{b}-\nabla_{b} \nabla_{a}$.

We say that $M$ is an anti-self-dual solution if

$$
\widetilde{\Psi}_{A^{\prime} B^{\prime} C^{\prime} D^{\prime}}=0, \quad \Phi_{a b}=0 ;
$$

i.e. if $M$ has anti-self-dual Weyl tensor and satisfies Einstein's equations with cosmological constant:

$$
R_{a b}=6 \Lambda g_{a b} .
$$


A curved, anti-self-dual space cannot be the complexification of a real, Lorentzian space-time, because in a Lorentzian space-time $\Psi_{A B C D}$ and $\tilde{\Psi}_{A^{\prime} B^{\prime} C^{\prime} D^{\prime}}$ are complex conjugates of each other, and so one of them cannot vanish without the other vanishing as well. An anti-self-dual space may, however, be the complexification of a positive definite Riemannian manifold.

We shall use some of the language of vector bundles. For example, let $P_{n}$ denote the $n$-dimensional complex projective space with homogeneous coordinates $z^{0}, z^{1}, \ldots, z^{n}$; let $L^{k}$ denote the holomorphic line bundle over $P_{n}$ with Chern number $k$; and let $\Gamma\left(P_{n}, L^{k}\right)$ denote the space of holomorphic sections of $L^{k}$. The elements of $\Gamma\left(P_{n}, L^{k}\right)$, i.e. the sections, are 'twisted functions' on $P_{n}$; one may think of them as functions of the homogeneous coordinates $z^{j}$ which are homogeneous of degree $k$. In fact, they have to be polynomials of degree $k$. So, for example,

$$
\Gamma\left(P_{1}, L^{0}\right) \cong C ;
$$

this is just Liouville's theorem (namely that a bounded analytic function of one complex variable has to be a constant). More generally,

$$
\Gamma\left(P_{1}, L^{k}\right)=C^{k+1}
$$

for $k \geqq 0$, since a homogeneous polynomial of degree $k$ in two complex variables depends on $k+1$ complex parameters.

\section{The Space-Time/Twistor Space Correspondence}

This section is devoted to a description of the correspondence between antiself-dual space-times and certain complex manifolds called deformed twistor spaces. First, let us take an anti-self-dual space $M$ and construct the corresponding twistor space $\mathscr{T}$. In brief, the points of $\mathscr{T}$ correspond to certain complex 2 -surfaces in $M$ called $\alpha$-surfaces. An $\alpha$-surface $Z$ is a 2 -surface in $M$ with the following property: there exists a primed spinor field $\pi_{A^{\prime}}$ on $Z$ such that each vector tangent to $Z$ has the form $\lambda^{A} \pi^{A^{\prime}}$, for some spinor $\lambda^{A}$. It follows from this definition that an $\alpha$-surface is totally null: any two of its tangent vectors are orthogonal. For if $v^{a}=\lambda^{A} \pi^{A^{\prime}}$ and $u^{a}=\mu^{A} \pi^{A^{\prime}}$ are two tangent vectors, then clearly $v_{a} u^{a}=0$, since $\pi_{A^{\prime}} \pi^{A^{\prime}}=0$.

Proposition [1] A necessary and sufficient condition for the existence of a threecomplex parameter family of $\alpha$-surfaces in $M$ is that $\widetilde{\Psi}_{A^{\prime} B^{\prime} C^{\prime} D^{\prime}}$ should vanish, i.e. that the Weyl tensor of $M$ be anti-self-dual.

From now on, let us suppose that $M$ satisfies $\widetilde{\Psi}_{A^{\prime} B^{\prime} C^{\prime} D^{\prime}}=0$, and let $\mathscr{T}$ denote the space of $\alpha$-surfaces in $M$. In view of the Proposition, $\mathscr{T}$ will, in some sense, be three-complex-dimensional. However, it may fail to be a Hausdorff manifold, owing to the global structure of $M$. As we are, at the moment, only interested in the local geometry of $M$, let us avoid such global problems. To be specific, let us take $M$ to be sufficiently small and convex to ensure that $\mathscr{T}$ is a complex manifold with topology $R^{4} \times S^{2}$. Every point in an anti-self-dual space possesses such a 'nice' neighbourhood. 
$\mathscr{T}$ is called the twistor space corresponding to $M$ (in the literature, it is usually referred to as projective twistor space, but we shall omit the word 'projective'). Each twistor $\mathbf{Z}$ in $\mathscr{T}$ corresponds to an $\alpha$-surface $Z$ in $M$. It is possible to choose the spinor field $\pi_{A^{\prime}}$ so that it is covariantly constant over $Z$,

$$
\pi^{B^{\prime}} \nabla_{B B^{\prime}} \pi_{A^{\prime}}=0
$$

the integrability conditions for $(2.1)$ being precisely $\tilde{\Psi}_{A^{\prime} B^{\prime} C^{\prime} D^{\prime}}=0$ (to see this, operate on (2.1) with $\pi^{C^{\prime}} \nabla^{B}{ }_{C^{\prime}}$ and use the Ricci identities (1.8)). Notice that the $\alpha$-surface $Z$ determines $\pi_{A^{\prime}}$ up to proportionality; this is obvious from its definition.

The complex manifold structure of $\mathscr{T}$ depends only on the conformal structure of $M$ : if one replaces the metric $g_{a b}$ on $M$ by $\Omega g_{a b}$, with $\Omega \neq 0$, then $\mathscr{T}$ does not change. So we need to introduce some additional, local structure on $\mathscr{T}$ which contains information about the metric and not just the conformal metric. This local structure consists of a pair of differential forms $\tau$ and $\rho$ on $\mathscr{T}$. The idea is that $\tau$ will contain information about $e_{A^{\prime} B^{\prime}}$ and $\rho$ information about $e_{A B}$, so that together they will determine the metric $g_{a b}$. To see what $\tau$ and $\rho$ are, we shall use a generalization of an unpublished argument due to Penrose.

Let $\mathbf{Q}$ be a vector at the point $\mathbf{Z}$ in $\mathscr{T}$. If $\varepsilon$ is an infinitesimal parameter, then one may think of $\varepsilon \mathbf{Q}$ as joining the point $\mathbf{Z}$ to a neighbouring point $\mathbf{Y}$ in $\mathscr{T}$. See Fig. 1. Let $v^{a}$ be a vector field on the $\alpha$-surface $Z$, such that $\varepsilon v^{a}$ is a connecting vector joining $Z$ to the neighbouring $\alpha$-surface $Y$. The fact that $\varepsilon v^{a}$ is a connecting vector implies that the Lie bracket of $v^{a}$ and $\lambda^{B} \pi^{B^{\prime}}$ vanishes for all $\lambda^{B}$ :
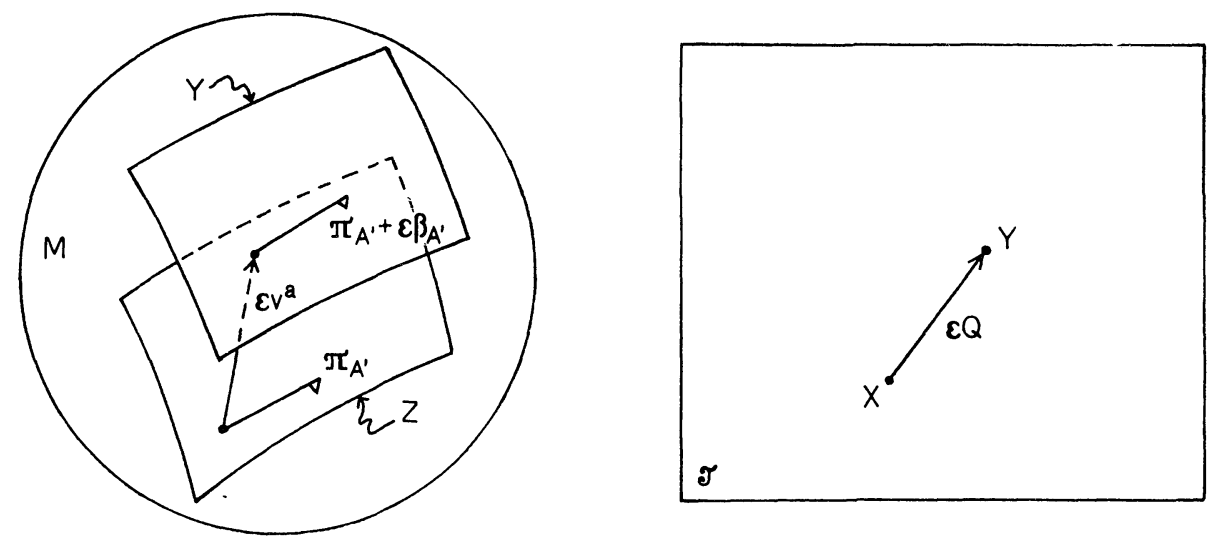

Fig. 1

$$
\lambda^{B} \pi^{B^{\prime}} \nabla_{B B^{\prime}} v^{A A^{\prime}}-v^{B B^{\prime}} \nabla_{B B^{\prime}} \lambda^{A} \pi^{A^{\prime}}=0 .
$$

From this it follows that

$$
\pi_{A^{\prime}} \lambda^{B} \pi^{B^{\prime}} \nabla_{B B^{\prime}} v^{A A^{\prime}}=\lambda^{A} \beta^{A^{\prime}} \pi_{A^{\prime}},
$$

where $\beta_{A^{\prime}}=v^{b} \nabla_{b} \pi_{A^{\prime}}$. By using the spinor Ricci identities $(1.7,1.8)$, one may easily show that the field $\beta_{A^{\prime}}(x)$ on $Z$ satisfies the transport equation

$$
\lambda^{B} \pi^{B^{\prime}} \nabla_{B B^{\prime}} \beta_{A^{\prime}}=-i \lambda^{B} \pi^{B^{\prime}} P_{A B A^{\prime} B^{\prime}} \alpha^{A},
$$


where $P_{a b}=-\frac{1}{2} R_{a b}+\frac{1}{12} R g_{a b}=\Phi_{a b}-\Lambda g_{a b}$ and $\alpha^{A}=i v^{A A^{\prime}} \pi_{A^{\prime}}$. From (2.2) it follows that the field $\alpha^{A}$ satisfies

$$
\lambda^{B} \pi^{B^{\prime}} \nabla_{B B^{\prime}} \alpha^{A}=-i \lambda^{A} \pi^{B^{\prime}} \beta_{B^{\prime}} .
$$

The pair $\left(\alpha^{A}, \beta_{A^{\prime}}\right)$ represents the vector $\mathbf{Q}$; this is called the local twistor representation [13]. The Eqs. (2.3) and (2.4) are the equations of local twistor transport and express the requirement that $\mathbf{Q}$ be 'constant over $\alpha$-surfaces'.

The representation has some freedom in it, since $\kappa\left(\alpha^{A}, \beta_{A^{\prime}}\right)$, where $\kappa$ is a nonzero constant, represents the same vector $\mathbf{Q}$. So $\left(\alpha^{A}, \beta_{A^{\prime}}\right)$ should be thought of as 'homogeneous coordinates' for $\mathbf{Q}$. Notice also that the pair $\left(0, \pi_{A^{\prime}}\right)$ corresponds to the zero vector $\mathbf{Q}=0$ (since $\alpha^{A}=0, \beta_{A^{\prime}}=\pi_{A^{\prime}}$ implies that $Y=Z$, and that the spinor $\pi_{A^{\prime}}$ is unchanged up to proportionality).

We shall now define the forms $\tau$ and $\rho$ on $\mathscr{T}$, beginning with $\tau$, which is a 1 -form. If $Q$, represented by $\left(\alpha^{A}, \beta_{A^{\prime}}\right)$ is any vector in $\mathscr{T}$, then by definition

$$
\tau(Q)=e^{A^{\prime} B^{\prime}} \pi_{A^{\prime}} \beta_{B^{\prime}}
$$

If $\tau$ is to be well-defined, the scalar on the right hand side of (2.5) must be constant over $\alpha$-surfaces, in other words must be annihilated by the differential operator $\lambda^{A} \pi^{A^{\prime}} \nabla_{A A^{\prime}}$; for otherwise $\tau$ will not correspond to a differential form on $\mathscr{T}$. From (2.3) we see that

$$
\lambda^{C} \pi^{C^{\prime}} \nabla_{C C^{\prime}}\left(e^{A^{\prime} B^{\prime}} \pi_{A^{\prime}} \beta_{B^{\prime}}\right)=i \lambda^{B} \pi^{B^{\prime}} \alpha^{A} \pi^{A^{\prime}} \Phi_{A B A^{\prime} B^{\prime}},
$$

so $\tau$ is well-defined if and only if $\Phi_{a b}=0$. From now on let us assume, therefore, that $M$ is an anti-self-dual solution, so that both $\widetilde{\Psi}_{A^{\prime} B^{\prime} C^{\prime} D^{\prime}}=0$ and $\Phi_{a b}=0$ hold.

Actually, strictly speaking, $\tau$ is not a form on $\mathscr{T}$, since it is homogeneous of degree two (one from the $\pi_{A^{\prime}}$ and one from the $\beta_{B^{\prime}}$ ). A 'pure' form would be independent of the scaling of $\pi_{A^{\prime}}$ and $\beta_{B^{\prime}}$, i.e. would be homogeneous of degree zero. So $\tau$ is really a 'twisted' form. We shall nevertheless continue to call $\tau$ a 1 -form, homogeneous of degree two.

Next we must define the form $\rho$, which is a 3 -form, homogeneous of degree four. So let $Q_{j}, j=1,2,3$, be three vectors in $\mathscr{T}$, represented by $\left(\alpha_{j}{ }_{j}, \beta_{j A^{\prime}}\right)$. Then $\rho\left(Q_{1}, Q_{2}, Q_{3}\right)$ is defined by taking

$$
\rho_{123}=\frac{1}{2}\left(e^{A^{\prime} B^{\prime}} \pi_{A^{\prime}} \beta_{1 B^{\prime}}\right)\left(e_{A B} \alpha_{2}^{A} \alpha_{3}^{B}\right)
$$

and skew-symmetrizing it over 1,2, 3. In other words,

$$
\rho\left(Q_{1}, Q_{2}, Q_{3}\right)=\frac{1}{6}\left(\rho_{123}-\rho_{132}+\rho_{312}-\rho_{321}+\rho_{231}-\rho_{213}\right) .
$$

Equations (2.3) and (2.4) imply that $\rho$ is well-defined, i.e. that $\lambda^{A} \pi^{A^{\prime}} \nabla_{A A^{\prime}} \rho\left(Q_{1}, Q_{2}\right.$, $\left.Q_{3}\right)=0$.

What has been shown so far may be summarized as follows. Let $M$ be a (sufficiently small) anti-self-dual solution. Then the corresponding projective twistor space $\mathscr{T}$ is a three-dimensional complex manifold, equipped with a 1 -form $\tau$ homogeneous of degree 2 and a 3 -form $\rho$ homogeneous of degree 4 . Furthermore, $\tau \wedge d \tau=2 \Lambda \rho$, where $24 \Lambda$ is the scalar curvature of $M$ (this is proved in the Appendix). 
As an example, let us take $M$ to be conformally flat, with metric

$$
\begin{aligned}
& g_{a b}=\Omega^{2} \eta_{a b}, \\
& \eta_{a b}=\operatorname{diag}\left(\begin{array}{llll}
1 & -1 & -1 & -1
\end{array}\right),
\end{aligned}
$$

and construct the corresponding twistor space $T$. Let the function $\Omega$ be given by

$$
\Omega^{-1}=1-\frac{1}{2} \Lambda \eta_{a b} x^{a} x^{b}
$$

then the space-time satisfies Einstein's equations $R_{a b}=6 \Lambda g_{a b}$, as required. Let $x^{A A^{\prime}}$ be the standard Minkowski spinor translation of $x^{a}[13]$.

Each $\alpha$-surface in $M$ is the solution space of an equation of the form

$$
\omega^{A}=i x^{A A^{\prime}} \pi_{A^{\prime}}
$$

for some fixed $\omega^{A}$ and $\pi_{A^{\prime}} \neq 0[13,1]$. Thus our 'flat' twistor space $T$ is a subset of the complex projective space $P_{3}$ :

$$
T=\left\{\left(\omega^{A}, \pi_{A^{\prime}}\right) \mid \pi_{A^{\prime}} \neq 0\right\} / \sim
$$

where $\sim$ is the equivalence relation of proportionality. [In other words, $\left(\omega^{A}, \pi_{A^{\prime}}\right)$ and $\left(\kappa \omega^{A}, \kappa \pi_{A^{\prime}}\right)$, with $\kappa \neq 0$, are equivalent.] Thus the space $T$ is $P_{3}$ with the line $\pi_{A^{\prime}}=0$ deleted.

A vector $Q$ in $T$ has the form

$$
Q=W^{A} \frac{\partial}{\partial \omega^{A}}+P_{A^{\prime}} \frac{\partial}{\partial \pi_{A^{\prime}}}
$$

in terms of our coordinates on $T$. We need to relate the representation $\left(W^{A}, P_{A^{\prime}}\right)$ of $Q$ to the local twistor representation $\left(\alpha^{A}, \beta_{A^{\prime}}\right)$ introduced earlier. In order to do this, we use eq. (2.8) and the expression

$$
\nabla_{A A^{\prime}} \pi_{B^{\prime}}=\partial \pi_{B^{\prime}} / \partial x^{A A^{\prime}}-\Omega \Lambda x_{A B^{\prime}} \pi_{A^{\prime}}
$$

(cf. [13], 1.19a) for the covariant derivative of $\pi_{A^{\prime}}$. A simple calculation yields

$$
\begin{aligned}
\alpha^{A} & =W^{A}-i x^{A A^{\prime}} P_{A^{\prime}}, \\
\beta_{A^{\prime}} & =\Omega P_{A^{\prime}}+i \Omega \Lambda x_{A A^{\prime}} W^{A} .
\end{aligned}
$$

In (2.12), the indices of $x_{A A^{\prime}}$ have been lowered using the flat-space Levi-Civita symbols $\varepsilon_{A B}$ and $\varepsilon_{A^{\prime} B^{\prime}}$ (that is to say, $\varepsilon_{01}=-\varepsilon_{10}=1, \varepsilon_{00}=\varepsilon_{11}=0$, and similarly for $\left.\varepsilon_{A^{\prime} B^{\prime}}\right)$. The curved-space forms $e$ and $e^{\prime}$ are given by

$$
e_{A B}=\Omega \varepsilon_{A B}, \quad e_{A^{\prime} B^{\prime}}=\Omega \varepsilon_{A^{\prime} B^{\prime}} .
$$

Now we can define the forms $\tau$ and $\rho$, by substituting (2.11) and (2.12) into (2.5) and (2.6). For example,

$$
\begin{aligned}
\pi_{A^{\prime}} \beta^{A^{\prime}} & =e^{A^{\prime} B^{\prime}} \pi_{A^{\prime}} \beta_{B^{\prime}} \\
& =\Omega^{-1} \varepsilon^{A^{\prime} B^{\prime}} \pi_{A^{\prime}}\left(\Omega P_{B^{\prime}}+i \Omega \Lambda x_{A B^{\prime}} W^{A}\right) \\
& =\pi_{A^{\prime}} P^{A^{\prime}}+\Lambda \omega_{A} W^{A}
\end{aligned}
$$


and so

$$
\tau=\pi_{A^{\prime}} d \pi^{A^{\prime}}+\Lambda \omega_{A} d \omega^{A} .
$$

A similar calculation gives

$$
\rho=\frac{1}{2} \pi_{A^{\prime}} d \pi^{A^{\prime}} \wedge d \omega_{A} \wedge d \omega^{A}+\frac{1}{2} \omega_{A} d \omega^{A} \wedge d \pi_{A^{\prime}} \wedge d \pi^{A^{\prime}}
$$

Remarks. (i) Notice that $\tau \wedge d \tau=2 \Lambda \rho$.

(ii) A point $x$ in space-time is characterized by the set of all $\alpha$-surfaces through it. This set is a compact holomorphic curve $\mathbf{x}$ (intrinsically a Riemann sphere) in $P T[1]$.

Let us now tackle the converse problem: suppose we are given $\mathscr{T}$ with its differential forms and asked to construct an anti-self-dual space $M$. The procedure for doing so is a generalization of that in references $[1,14]$.

The basic idea is to use remark (ii) above. Assume that $\mathscr{T}$ has topology $R^{4} \times S^{2}$ and look for holomorphic curves in $\mathscr{T}$ which are intrinsically Riemann spheres and which 'wrap once around $\mathscr{T}$ ' (i.e. which belong to the generating homology class of $\mathscr{T}$ ). We require that $\mathscr{T}$ satisfy the following condition.

There is precisely a four-complex-parameter family of such curves, and the

$$
\text { normal bundle of each curve is isomorphic to } L^{1} \oplus L^{1} \text {. }
$$

[The normal bundle $N_{\mathbf{x}}$ of a submanifold $\mathbf{x}$ in $\mathscr{T}$ is, roughly speaking, the bundle of vectors transverse to $\mathbf{x} . L^{1}$ denotes the line bundle over $\mathbf{x}$ which has Chern number 1 (cf. Sect. 1).]

The condition (2.15) is a tricky one to have to impose. Fortunately, results of Kodaira [15] show that if we begin with flat twistor space (2.9), which satisfies (2.15), and deform its complex structure, then the property (2.15) is preserved, provided that the deformation is sufficiently small. This is proved in $[16,14]$; the proofs there are in the context of the special case where $\Lambda=0$, but they carry over unchanged to the present, more general, case. The restriction to sufficiently small deformations corresponds precisely to the condition imposed previously that the space-time $M$ be 'sufficiently local'. In the local problem, therefore, condition (2.15) will automatically be satisfied.

Given that (2.15) is satisfied, it is an easy matter to construct the space $M$ : let $M$ be the four-complex-dimensional space of holomorphic curves in $\mathscr{T}$. Each point $x$ in $M$ corresponds to a holomorphic curve $\mathbf{x}$ in $\mathscr{T}$. Let $N_{\mathbf{x}}$ be the normal bundle of $\mathbf{x}$ in $\mathscr{T}$. By (2.15), $N_{\mathbf{x}}$ is isomorphic to $L^{1} \oplus L^{1}$ (considered as a bundle over the Riemann Sphere $\mathbf{x}$ ). A tangent vector $v$ at $x \in M$ corresponds to a section $\mathbf{v}$ of $N_{\mathbf{x}}$ :

$$
T_{\chi}(M) \cong \Gamma\left(\mathbf{x}, N_{\mathbf{x}}\right)
$$

[Recall from Eq. (1.11) that $\Gamma\left(P_{1}, L^{1}\right) \cong C^{2}$, so

$$
\Gamma\left(\mathbf{x}, N_{\mathbf{x}}\right) \cong \Gamma\left(P_{1}, L^{1} \oplus L^{1}\right) \cong C^{2} \oplus C^{2} \cong C^{4},
$$

as one would expect for the tangent space $T_{x}(M)$.] 
We now define a conformal structure on $M$ by

$v$ is a null vector $\Leftrightarrow \mathbf{v}$ vanishes somewhere on $\mathbf{x}$.

(It is easy to check that this does indeed define a conformal structure $[1,14]$.) Through each point $\mathbf{z} \in \mathscr{T}$ there passes a 2-parameter family of holomorphic curves, corresponding to a 2 -surface $Z$ in $M$. From the definition (2.17) it follows immediately that $Z$ is totally null (in fact, it is an $\alpha$-surface). Hence we deduce from the Proposition in Sect. 2 that the conformal structure we have defined is anti-self-dual (i.e. $\tilde{\Psi}_{A^{\prime} B^{\prime} C^{\prime} D^{\prime}}=0$ ).

To define a metric on $M$ necessitates the use of the additional structure on twistor space. We proceed as follows. Let $V$ be the subbundle of the tangent bundle of $\mathscr{T}$ consisting of those vectors which are annihilated by $\tau$. In other words, $\xi \in V$ if and only if $\tau(\xi)=0$. Suppose that $\mathbf{x}$ is a holomorphic curve transverse to $V$ (i.e. the tangent vector of the curve $\mathbf{x}$ does not lie in $V$ ). We shall restrict our attention to space-time points for which this is the case; the metric is not defined at other points. With this restriction, it follows that $V_{\mathbf{x}}$ (i.e. $V$ restricted to $\mathbf{x}$, a 2-dimensional vector bundle over $\mathbf{x}$ ) is naturally isomorphic to the normal bundle $N_{\mathbf{x}}$ of $\mathbf{x}$. Thus a vector $v$ in $T_{\mathbf{x}}(M)$ corresponds to a section $\mathbf{v}$ of $V_{\mathbf{x}}$.

Let $x$ be a point of $M$ and define two 2-dimensional spin-spaces $S$ and $S^{\prime}$ at $\mathbf{x}$ by

$$
\begin{aligned}
S & =\Gamma\left(\mathbf{x}, V_{\mathbf{x}} \otimes L^{-1}\right), \\
S^{\prime} & =\Gamma\left(\mathbf{x}, L^{1}\right) .
\end{aligned}
$$

Since $V_{\mathbf{x}} \otimes L^{-1} \cong\left(L^{1} \oplus L^{1}\right) \otimes L^{-1} \cong L^{0} \oplus L^{0}$, it follows from (1.10) that $S \cong C^{2}$; and (1.11) implies that $S^{\prime} \cong C^{2}$ as well. It is easily seen that $S \otimes S^{\prime}$ is naturally isomorphic to $\Gamma\left(\mathbf{x}, V_{\mathbf{x}}\right)$ and hence to $T_{x}(M)$; and that a vector $v$ in $T_{\mathbf{x}}(M)$ is null if and only if it is the tensor product of a primed and an unprimed spinor, i.e. if and only if $v=\alpha \xi$, where $\alpha \in S^{\prime}$ and $\xi \in S$.

We now have to define the forms $e_{A B}$ and $e_{A^{\prime} B^{\prime}}$ on $S$ and $S^{\prime}$ respectively. Let $\xi$ and $\mu$ be two elements of $S$. Thus $\xi$ and $\mu$ are, in view of (2.18a), vector fields on $\mathbf{x}$ which are homogeneous of degree -1 (tensoring with $L^{-1}$ has the effect of reducing the homogeneity from 0 to -1 : cf. Sect. 1). Let $v$ be any other vector field on $\mathbf{x}$, such that $\tau(v) \neq 0$. We define the 2 -form $e_{A B}$ on $S$ by saying that the scalar $e(\xi, \mu)$ is given by

$$
e(\xi, \mu) \tau(v)=-6 \rho(v, \xi, \mu)
$$

The quantity $e(\xi, \mu)$ is independent of $v$, since $\tau$ annihilates both $\xi$ and $\mu$, and so $[\tau(v)]^{-1} \rho(v, \xi, \mu)$ is invariant under the transformation $v \mapsto a v+b \xi+c \mu$. Furthermore, $e(\xi, \mu)$ is homogeneous of degree zero in $\xi$ and $\mu$, and so by Liouville's theorem (1.10) it is a constant.

Next let us define $e_{A^{\prime} B^{\prime}}$ on $S^{\prime}$. Let $\alpha$ and $\beta$ be two elements of $S^{\prime}$. So, by $(2.18 \mathrm{~b})$, $\alpha$ and $\beta$ are functions on $\mathbf{x}$, homogeneous of degree 1 . Consider the 1 -form $\alpha d \beta-\beta d \alpha$ on $\mathbf{x}$. Since $\mathbf{x}$ is a one-dimensional space, this form must be a multiple of $\left.\tau\right|_{\mathbf{x}}$, the form 1-form $\tau$ restricted to the curve $\mathbf{x}$. We define $e_{A^{\prime} B^{\prime}}$ on $S^{\prime}$ by saying 
that the scalar $e^{\prime}(\alpha, \beta)$ is given by

$$
\alpha d \beta-\beta d \alpha=\left.e^{\prime}(\alpha, \beta) \tau\right|_{\mathbf{x}} .
$$

Our assumption that the curve $\mathbf{x}$ is transverse to $V$ ensures that $\left.\tau\right|_{\mathbf{x}} \neq 0$, so $e^{\prime}(\alpha, \beta)$ is well-defined. Since both $\alpha d \beta-\beta d \alpha$ and $\left.\tau\right|_{\mathbf{x}}$ are homogeneous of degree two, $e^{\prime}(\alpha, \beta)$ is homogeneous of degree zero, and so, by Liouville's theorem again, it is a constant.

In Sect. 3 it is shown that the metric $g_{a b}=e_{A B} e_{A^{\prime} B^{\prime}}$ is an anti-self-dual solution, and that its scalar curvature $R=24 \Lambda$ is given by the formula

$$
\tau \wedge d \tau=2 \Lambda \rho \text {. }
$$

This establishes our main theorem.

Theorem. There is a one-to-one correspondence between

(i) (sufficiently local) anti-self-dual solutions with scalar curvature $R=24 \Lambda$; and

(ii) (sufficiently small) deformations of flat projective twistor space which preserve the 1-form $\tau$ (homogeneous of degree 2 ) and the 3-form $\rho$ (homogeneous of degree 4 ); and where $\tau \wedge d \tau=2 \Lambda \rho$.

Remarks. (i) The bundle $V \otimes L^{-1}$ is a 2-dimensional complex vector bundle over $\mathscr{T}$, and is trivial over every holomorphic curve $\mathbf{x}$. Therefore it corresponds to an anti-self-dual Yang-Mills field in the space-time $M$ [17]. This means that that we have a 2-dimensional vector bundle $E$ over $M$ and a connection on $E$ with anti-self-dual curvature. In fact, $E$ is precisely the unprimed spin-bundle $S$, and the connection on it is the natural one induced by the metric $g$ on $M$. This observation is due to Singer [4].

(ii) If $\Lambda=0$, then $d \tau \wedge \tau=0$, which means that $V$ is an integrable distribution on $\mathscr{T}$. In this case, $\mathscr{T}$ has the structure of a holomorphic bundle over the Riemann sphere $P_{1}$, each fibre being an integral surface of $V[1]$.

(iii) The manifold $\mathscr{T}$ and the forms $\tau$ and $\rho$ are not subject to any differential equations, unlike the space-time metric, which of course satisfies the anti-self-dual Einstein equations. So these nonlinear differential equations disappear when one goes from the $M$-picture of the $\mathscr{T}$-picture.

(iv) There are modified versions of the Theorem which describe solutions satisfying additional conditions: for example, that $M$ be the complexification of a compact, positive definite space (cf. $[4,7]$ ).

\section{Details of the Construction}

This section is devoted to filling in some of the details of the construction which takes one from a deformed twistor space $\mathscr{T}$ to an anti-self-dual metric. The arguments will involve local homogeneous coordinates $Z^{\alpha}=\left(\omega^{A}, \pi_{A^{\prime}}\right)$ on $\mathscr{T}$ ('homogeneous' meaning that $Z^{\alpha}$ and $\lambda Z^{\alpha}$, with $\lambda \neq 0$, represent the same point of $\mathscr{T}$ ). Of course, these coordinates only cover a local coordinate patch $U$ in $\mathscr{T}$, unlike the situation in flat twistor space $T$, where they cover the whole space. It is always possible to choose the coordinates so that $\tau$ and $\rho$ are given by the 
flat-space expressions (2.13) and (2.14), and we shall suppose that such a choice has been made.

The holomorphic curves in $\mathscr{T}$ are given by an expression of the form

$$
Z^{\alpha}=h^{\alpha}\left(x^{b}, \zeta_{B^{\prime}}\right)
$$

in the patch $U$. Here the $x^{b}$ are four complex parameters which label the different curves, and therefore serve as coordinates on $M$, whereas the two parameters $\zeta_{B^{\prime}}$ serve as homogeneous coordinates on each holomorphic curve (a 'holomorphic curve', remember, is intrinsically a Riemann sphere). For the sake of consistency, the functions $h^{\alpha}$ are required to be homogeneous of degree one in $\zeta_{B^{\prime}}$.

A vector $v$ at $x \in M$ corresponds in $\mathscr{T}$ to a vector field $\mathbf{v}$ on the curve $\mathbf{x}$ (think of $\mathbf{v}$ as a connecting vector joining $\mathbf{x}$ to a neighbouring curve). Let $v^{a}$ be the components of $v$ with respect to the coordinate basis $\partial_{a}=\partial / \partial x^{a}$. Then clearly the vector field $\mathbf{v}$ will have the form

$$
\mathbf{v}=\left\{v^{b} D_{b} h^{\alpha}(x, \zeta)\right\} \frac{\partial}{\partial Z^{\alpha}}
$$

where $D_{b}$ is the operator

$$
D_{b}=\partial_{b}+f_{b A^{\prime}}(x, \zeta) \frac{\partial}{\partial \zeta_{A^{\prime}}} .
$$

The $\partial / \partial \zeta$ term in (3.3) corresponds to a vector field tangent to $\mathbf{x}$, so we may add it in without altering the fact that $\mathbf{v}$ is a connecting vector. The functions $f_{b A^{\prime}}$ are chosen so that $\tau(\mathbf{v})=0$; in other words, so that $\mathbf{v}$ lies in the vector bundle $V$ (Fig. 2). The remaining freedom in $f_{b A^{\prime}}$ is $f_{b A^{\prime}} \mapsto f_{b A^{\prime}}+f_{b} \zeta_{A^{\prime}}$, i.e. $\mathbf{v} \mapsto \mathbf{v}+\left(v^{b} f_{b}\right) r$, where $r=\zeta_{A^{\prime}} \partial / \partial \zeta_{A^{\prime}}$ is the Euler homogeneity operator. We should think of this vector $Y$ as being equivalent to zero in $\mathscr{T}$; this corresponds to the fact that $Z^{\alpha}$ are homogeneous coordinates on $\mathscr{T}$. This remaining ambiguity may be removed by imposing the condition

$$
v \text { is } n u l l \Leftrightarrow \mathbf{v} \text { vanishes somewhere on } \mathbf{x} .
$$
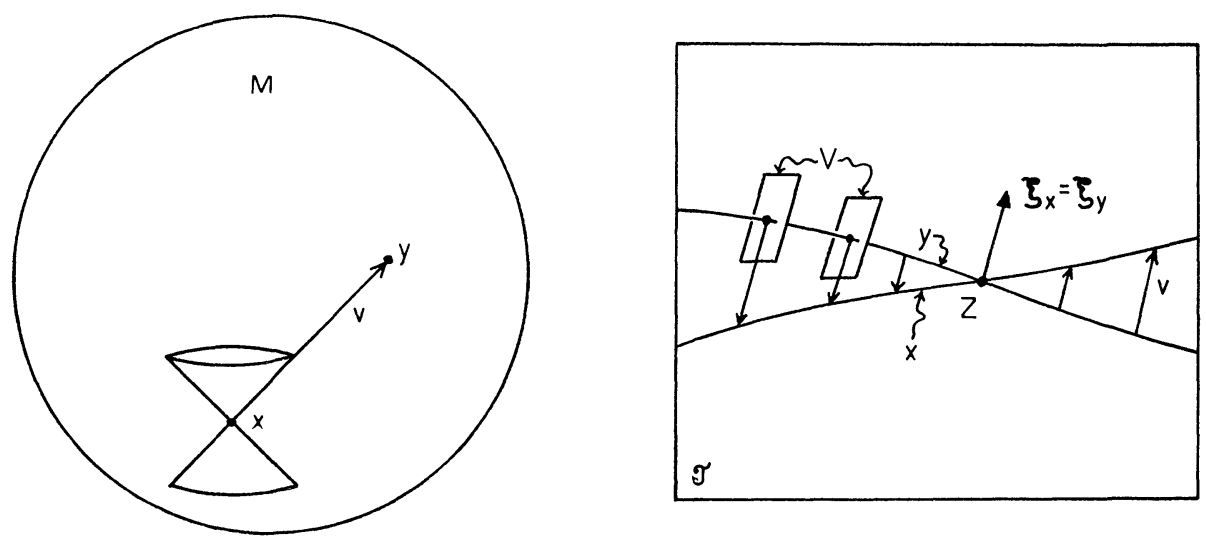

Fig. 2 
Thus if $v$ is null, then $\mathbf{v}$ vanishes at (say) $\zeta_{A^{\prime}}=\gamma_{A^{\prime}}$, and we can factorize $\mathbf{v}$ :

$$
\mathbf{v}(x, \zeta)=\left(\gamma^{A^{\prime}} \zeta_{A^{\prime}}\right) \xi(x, \zeta)
$$

Here $\gamma^{A^{\prime}} \zeta_{A^{\prime}}$, being a function homogeneous of degree one in $\zeta_{A^{\prime}}$, is an element of $\Gamma\left(\mathbf{x}, L^{1}\right)$ (cf. Sect. 1); in other words, is an element of the primed spin-space $S^{\prime}$. And $\xi$ is a vector field on $\mathbf{x}$, homogeneous of degree zero; in other words, $\xi$ is an element of the unprimed spin-space $S=\Gamma\left(\mathbf{x}, V_{\mathbf{x}} \otimes L^{-1}\right)$. Equation (3.5) is simply the $\mathscr{T}$-picture version of the statement that a vector $v$ is null if and only if it is the tensor product of a primed and an unprimed spinor.

We shall now use this local coordinate formalism to prove (as was promised in Sect. 2) that the metric $g_{a b}$ constructed from $\mathscr{T}, \tau$ and $\rho$ is indeed an anti-self-dual solution. The idea of the proof, which follows [1], is to define a connection $\nabla$ on $M$, then show that $\nabla$ is the unique connection determined by $g_{a b}$, and finally demonstrate that $\nabla$ is anti-self-dual.

To determine $\nabla$, it suffices to specify what is meant by parallel propagation of spinors along curves in $M$. In fact, it is sufficient, by linearity, to specify how to propagate spinors in null directions. Suppose, therefore, that $x$ and $y$ are neighbouring null-separated points in $M$; let $v$ be the null vector joining $x$ to $y$ (Fig. 2). In $\mathscr{T}$, the holomorphic curves $\mathbf{x}$ and $\mathbf{y}$ are joined by the vector field $\mathbf{v}$. Since $v$ is null, the curves $\mathbf{x}$ and $\mathbf{y}$ intersect at some point $\mathbf{Z}$. The vector field $\mathbf{v}$ defines a natural map $\sigma$ from $\mathbf{x}$ to $\mathbf{y}$, and induces a natural map $\tilde{\sigma}$ from the line bundle $L^{1}$ over $\mathbf{x}$ to the line bundle $L^{1}$ over $\mathbf{y}$ (their fibres coincide at $\mathbf{Z}$ ). So there is a natural way of propagating a primed spinor at $x, \alpha_{x} \in \Gamma\left(\mathbf{x}, L^{1}\right)$, to $y$ : put $\alpha_{y}=$ $\tilde{\sigma} \cdot \alpha_{x} \cdot \sigma^{-1}$.

Next, suppose that $\xi_{x} \in \Gamma\left(\mathbf{x}, V_{\mathbf{x}} \otimes L^{-1}\right)$ is an unprimed spinor at $x$; we want to define $\xi_{y}$. The value of $\xi_{y}$ at $\mathbf{Z} \in \mathbf{y}$ determines its value everywhere on $\mathbf{y}$, so we may specify $\xi_{y}$ by saying that $\xi_{x}$ and $\xi_{y}$ should be equal at $\mathbf{Z}=\mathbf{x} \cap \mathbf{y}$ (cf. Fig. 2). This completes the definition of $\nabla$.

Lemma 1. $\nabla$ is torsion-free.

Proof. It suffices to prove that if $v_{0}$ and $v_{1}$ are two null vector fields on $M$ which commute with each other, then the vector field

$$
w=\nabla_{0} v_{1}-\nabla_{1} v_{0}
$$

vanishes $\left(\nabla_{A}\right.$ denotes the covariant derivative along $\left.v_{A}\right)$. Let us work in a local coordinate patch $U$, and use the notation introduced earlier in this section. Thus the connecting vector field $\mathbf{v}_{0}=\mathbf{v}_{0}{ }^{\alpha} \partial / \partial Z^{\alpha}$ on a holomorphic curve $\mathbf{x}$ is given by

$$
\mathbf{v}_{0}{ }^{\alpha}=v_{0}{ }^{b} D_{b} h^{\alpha}=\gamma_{0} \xi_{0}{ }^{\alpha},
$$

where $\gamma_{0}=\gamma_{0}{ }^{A^{\prime}}(x) \zeta_{A^{\prime}}$; and similarly for $v_{1}$.

If $\alpha$ and $\xi=\xi^{\alpha} \partial / \partial Z^{\alpha}$ are, respectively, a primed and an unprimed spinor field, their covariant derivatives along $v_{A}$ are given by

$$
\begin{aligned}
& \nabla_{A} \alpha=v_{A}{ }^{b} D_{b} \alpha, \\
& \nabla_{A} \xi=\left(v_{A}{ }^{b} D_{b} \xi^{\alpha}\right) \partial / \partial Z^{\alpha}+0\left(\gamma_{0}\right),
\end{aligned}
$$


where $0(\gamma)$ denotes a term which vanishes at $\gamma=0$. The vector field $\mathbf{w}=\mathbf{w}^{\alpha} \partial / \partial Z^{\alpha}$ on $\mathbf{x}$, corresponding to (3.6), is

$$
\mathbf{w}^{\alpha}=\left[v_{0}^{b} D_{b}, v_{1}^{c} D_{c}\right] h^{\alpha}+0\left(\gamma_{0} \gamma_{1}\right)
$$

this follows from (3.7), (3.8) and (3.9). Expanding the right hand side of (3.10) reveals that $\mathbf{w}$ is tangent to $\mathbf{x}$ at the two points $\gamma_{0}=0$ and $\gamma_{1}=0$ on $\mathbf{x}$; it follows that $\mathbf{w}$ is everywhere tangent to $\mathbf{x}$ and hence that the vector $w$ vanishes.

Having shown that $\nabla$ is torsion-free, we may now considerably simplify matters by restricting our attention to a single (generic) $\alpha$-surface $Y$ in $M$. Let $Y$ be a point of $\mathscr{T}$ and suppose that the local coordinates $Z^{\alpha}=\left(\omega^{A}, \pi_{A^{\prime}}\right)$ in $U$ are chosen so that the point $\mathbf{Y}$ has coordinates $\left(\omega^{A}, \pi_{A^{\prime}}\right)=\left(0, \gamma_{A^{\prime}}\right)$, where $\gamma_{A^{\prime}}$, is some fixed spinor. The two-parameter family of points $y$ on the $\alpha$-surface $Y$ correspond, in $\mathscr{T}$, to the two-parameter family of holomorphic curves $\mathbf{y}$ which pass through $\mathbf{Y}$. Choose the parameter $\zeta_{A^{\prime}}$ on each of the curves $\mathbf{y}$ in such a way that

$h^{\alpha}\left(y, \gamma_{B^{\prime}}\right)=\left(0, \gamma_{A^{\prime}}\right)$ for all $y \in Y$; and specify coordinates $y^{A}=\left(y^{0}, y^{1}\right)$ on $Y$ by saying that for points $y$ on $Y, h^{\alpha}$ has the form

$$
h^{\alpha}\left(y^{B}, \zeta_{B^{\prime}}\right)=\left(\gamma y^{A}, \zeta_{A^{\prime}}\right)+0\left(\gamma^{2}\right)
$$

where $\gamma=\gamma^{A^{\prime}} \zeta_{A^{\prime}}$.

Let $v_{0}$ and $v_{1}$ be the two coordinate vectors on $Y$, i.e. $v_{A}=\partial / \partial y^{A}$. Suppose that the functions $f_{b A^{\prime}}$ satisfy

$$
v_{A}^{b} f_{b A^{\prime}}=-\Lambda \gamma y_{A} \gamma_{A^{\prime}}
$$

then the vector field $\mathbf{v}_{A}=\mathbf{v}_{A}{ }^{\beta} \partial / \partial Z^{\beta}$ corresponding to $v_{A}$ is given by

$$
\mathbf{v}_{A}{ }^{\beta}=v_{A}{ }^{B} D_{B} h^{\beta}=\gamma\left(\varepsilon_{A}{ }^{B},-\Lambda y_{A} \gamma_{B^{\prime}}\right)+0\left(\gamma^{2}\right) \text {. }
$$

Equation (3.13) follows directly from (3.2), (3.3), (3.11) \& (3.12). One may easily check that $\tau\left(\mathbf{v}_{A}\right)=0$, and this justifies the choice (3.12).

A primed spinor field $\alpha$ on $Y$ is represented in the $\mathscr{T}$-picture by a linear function $\alpha=\alpha^{A^{\prime}}\left(y_{A}\right) \zeta_{A^{\prime}}$ of $\zeta$. Simple calculations now reveal that on the $\alpha$-surface $Y$ we have

$$
\begin{aligned}
e^{\prime}(\alpha, \beta) & =\varepsilon_{A^{\prime} B^{\prime}} \alpha^{A^{\prime}} \beta^{B^{\prime}}, \\
\nabla_{A} \alpha & =\left(v_{A}{ }^{b} \partial_{b} \alpha^{A^{\prime}}-\Lambda \gamma^{A^{\prime}} y_{A} \gamma_{B^{\prime}} \alpha^{B^{\prime}}\right) \pi_{A^{\prime}} .
\end{aligned}
$$

Similarly, an unprimed spinor field $\xi$ on $Y$ is represented in the $\mathscr{T}$-picture by a vector field $\xi=\xi^{B}\left(y_{A}\right) h_{B}$, where

$$
h_{B}=\frac{\partial}{\partial \omega^{B}}-\Lambda y_{B} \Upsilon_{A^{\prime}} \frac{\partial}{\partial \zeta_{A^{\prime}}}+0(\gamma) .
$$

And it turns out that

$$
\begin{aligned}
e(\xi, \mu) & =-\varepsilon_{A B} \xi^{A} \mu^{B}, \\
\nabla_{A} \xi & =\left(v_{A}{ }^{b} \partial_{b} \xi^{C}\right) h_{C}+g_{A} \Upsilon+0(\gamma),
\end{aligned}
$$

where the $g_{A}$ are functions where exact form does not concern us.

Lemma 2. $\nabla$ is a metric connection, i.e. $\nabla g=0$. 
Proof. It suffices (by linearity) to prove that $\nabla_{A} e^{\prime}=0$ and $\nabla_{A} e=0$ on $Y$, and these two results are immediate consequences of $(3.14,3.15)$ and $(3.17,3.18)$ respectively.

Lemma 3. The connection $\nabla$, acting on the unprimed spin-bundle $S$, is integrable over the $\alpha$-surface $Y$.

Proof. The result is obvious from the geometric definition of $\nabla$ : as the curve $\mathbf{x}$ varies through the fixed point $\mathbf{Z}$, the vector $\xi$ at $\mathbf{Z}$ remains fixed (see Fig. 2). Of course, the result can also be deduced from (3.18).

Lemma 4. $g$ is an anti-self-dual solution, i.e. it satisfies $\widetilde{\Psi}_{A^{\prime} B^{\prime} C^{\prime} D^{\prime}}=0, \Phi_{A B A^{\prime} B^{\prime}}=0$.

Proof. By construction, $M$ contains a 3-complex-parameter family of $\alpha$-surfaces (one for each point of $\mathscr{T}$ ). So the Proposition in Sect. 1 tells us that $\widetilde{\Psi}_{A^{\prime} B^{\prime} C^{\prime} D^{\prime}}=0$. Lemmas 1 and 2 imply that $\nabla$ is the unique connection determined by $g$ and Lemma 3 says that the equation

$$
\pi^{A^{\prime}} \nabla_{A A^{\prime}} \xi_{B}=0
$$

is integrable. Operating on (3.19) with $\pi^{B^{\prime}} \nabla_{B^{\prime}}^{A}$ and using the Ricci identities (1.5) yield

$$
\pi^{A^{\prime}} \pi^{B^{\prime}} \Phi_{A B A^{\prime} B^{\prime}}=0
$$

Since (3.20) holds for all $\pi^{A^{\prime}}$, and $\Phi_{A B A^{\prime} B^{\prime}}$ is symmetric in $A^{\prime} B^{\prime}$, it follows that $\Phi_{A B A^{\prime} B^{\prime}}=0$, as required.

Lemma 5. The scalar curvature of $g$ is $R=24 \Lambda$.

Proof. Let $\alpha=\alpha^{A^{\prime}} \pi_{A^{\prime}}$ be a primed spinor. From (3.15) it follows that

$$
\left[\nabla_{A}, \nabla_{B}\right] \alpha=-2 \Lambda \varepsilon_{A B} \gamma \gamma_{A^{\prime}} \alpha^{A^{\prime}}
$$

But we can also compute this commutator by using the Ricci identities (1.7) and (1.8), the fact that $\left[v_{A}, v_{B}\right]$ vanishes, and the fact that the spinor version of $v_{A}$ is $v_{A}{ }^{B B^{\prime}}=\gamma^{B^{\prime}} \varepsilon_{A}{ }^{B}$. We then obtain

$$
\left[\nabla_{A}, \nabla_{B}\right] \alpha=2(R / 24) e_{A B} \gamma \gamma_{A^{\prime}}, \alpha^{A^{\prime}}
$$

Comparing (3.21) and (3.22) and using (3.17) gives the desired result.

\section{Example: $P_{2}$}

In this section we shall see how the 'gravitational instanton' $P_{2}$, with the FubiniStudy metric $[10,11]$, may be constructed out of twistor data.

Let $\mathscr{T}$ be the flag manifold of $P_{2}$. In other words, each point $\mathbf{Z}$ of $\mathscr{T}$ is a pair $(p, l)$, where $p$ is a point of $P_{2}$ and $l$ is a line of $P_{2}$ containing $p$. Let us use homogeneous coordinates on $P_{2}$; thus $p$ has coordinates $p^{j}, l$ has coordinates $l_{j}$, and the incidence relation between $l$ and $p$ is $p^{j} l_{j}=0$. A holomorphic curve in $\mathscr{T}$ has the following form: let $L$ be a line in $P_{2}$ and $P$ a point in $P_{2}$ not on $L$; then the holomorphic curve in $\mathscr{T}$ consists of all pairs $(p, l)$, where $l$ passes through $P$ and where $\{p\}=l \cap L$. If $P^{j}$ and $L_{j}$ are the homogeneous coordinates of $P$ and $L$, then the 
equation of the curve is $L_{j} p^{j}=l_{j} p^{j}=l_{j} P^{j}=0$. Let us write $P^{j}=(\tilde{X}, \tilde{Y}, 1)$ and $L_{j}=(X, Y, 1)$, so that the parameters $x^{a}=(X, \tilde{X}, Y, \tilde{Y})$ serve as coordinates on the space $M$.

To compute the conformal structure of $M$ requires only some simple analytic geometry. What one finds is the following. If $x^{a}=(X, \tilde{X}, Y, \tilde{Y})$ and $x^{\prime a}=\left(X^{\prime}, \tilde{X}^{\prime}\right.$, $\left.Y^{\prime}, \tilde{Y}^{\prime}\right)$ are two points in $M$, then $x$ and $x^{\prime}$ are null-separated if and only if

$\left(1+Y^{*} \tilde{Y}^{*}\right) \Delta X \Delta \tilde{X}+\left(1+X^{*} \tilde{X}^{*}\right) \Delta Y \Delta \tilde{Y}-X^{*} \tilde{Y}^{*} \Delta Y \Delta \tilde{X}-Y^{*} \tilde{X}^{*} \Delta X \Delta \tilde{Y}=0$,

where $\Delta X=X^{\prime}-X, \quad X^{*}=\frac{1}{2}\left(X+X^{\prime}\right)$ etc.

It should be emphasized that (4.1) is the equation of the 'whole' null cone in $M$, and not just of the 'infinitesimal' null cones in the tangent bundle of $M$.

What we have done so far is already well-known $[4, p$. 438]. We now want to define a metric on $M$ by introducing forms $\tau$ and $\rho$ on $\mathscr{T}$. Let us take

$$
\begin{aligned}
& \tau=\frac{1}{2} l_{j} d p^{j}-\frac{1}{2} p^{j} d l_{j}, \\
& \rho=-\frac{1}{2} \tau \wedge d \tau .
\end{aligned}
$$

These forms determine a metric $g_{a b}$ compatible with the conformal structure (4.1). The computation of $g_{a b}$ is somewhat messy: what follows is a summary of its main steps.

The first step is to write the holomorphic curve $x$ corresponding to the point $x^{a}=(X, \tilde{X}, Y, \tilde{Y})$ in parametric form (cf. 3.1):

$$
\begin{aligned}
p^{j}(x, \zeta) & =\left(-Y \tilde{X} \zeta_{0^{\prime}}-(1+Y \tilde{Y}) \zeta_{1^{\prime}},(1+X \tilde{X}) \zeta_{0^{\prime}}+X \tilde{Y} \zeta_{1^{\prime}},-Y \zeta_{0^{\prime}}+X \zeta_{1^{\prime}}\right) \\
l_{j}(x, \zeta) & =\left(\zeta_{0^{\prime}}, \zeta_{1^{\prime}},-\tilde{X} \zeta_{0^{\prime}}-\tilde{Y} \zeta_{1^{\prime}}\right)
\end{aligned}
$$

It is easily checked that $p^{j} l_{j}=L_{j} p^{j}=P^{j} l_{j}=0$, as required.

Following $(3.2,3.3,3.4)$, one now finds the connecting vector field $\mathbf{v}_{a}$ on $\mathbf{x}$ corresponding to the coordinate vector $v_{a}=\partial / \partial x^{a}$ at $x \in M$. It turns out to be

$$
\mathbf{v}_{a}=J^{-1}\left(L_{k} \partial_{a} p^{k}\right) P^{j} \frac{\partial}{\partial p^{j}}+J^{-1}\left(P^{k} \partial_{a} l_{k}\right) L_{j} \frac{\partial}{\partial l_{j}},
$$

where $J=1+X \tilde{X}+Y \tilde{Y}$. It is obvious from (4.1) that the vectors $v_{a}$ are all null, so (cf. 3.5) one expects that there should exist objects $\Sigma_{B B^{\prime}}^{a}(x)$ and $h_{B}(x, \zeta)$ such that

$$
\Sigma^{a}{ }_{B B^{\prime}}, \mathbf{v}_{a}=\zeta_{B}, h_{B} .
$$

These $\Sigma^{a}{ }_{B B^{\prime}}$ are (up to a conformal factor) the Infeld-van der Waerden symbols (cf. Sect. 1). In fact, $h_{B}$ and $\Sigma_{B B^{\prime}}$ are given by

$$
\begin{aligned}
& h_{0}=p^{j} \frac{\partial}{\partial p^{j}}, \\
& h_{1}=-J^{-1} L_{j} \frac{\partial}{\partial l_{j}}, \\
& \Sigma_{00^{\prime}}^{0}=-X \tilde{Y},
\end{aligned}
$$




$$
\begin{aligned}
\Sigma^{0}{ }_{01^{\prime}} & =1+X \tilde{X}, \\
\Sigma^{1}{ }_{00^{\prime}} & =-(1+Y \tilde{Y}), \\
\Sigma^{1}{ }_{01^{\prime}} & =Y \tilde{X}, \\
\Sigma^{2}{ }_{11^{\prime}} & =\Sigma^{3}{ }_{00^{\prime}}=1,
\end{aligned}
$$

with all other components zero. If $\Sigma_{a}{ }^{B B^{\prime}}$ is the inverse of $\Sigma^{a}{ }_{B B^{\prime}}$, then the conformal metric (4.1) is indeed given by

$$
\Omega \equiv \varepsilon_{A B^{\prime}{ }^{\prime} B^{\prime}} \sum_{a}{ }^{A A^{\prime}} \Sigma_{b}{ }^{B B^{\prime}} d x^{a} d x^{b} .
$$

A primed spinor (i.e. an element of $S^{\prime}$ ) is an object of the form $\alpha^{A^{\prime}} \zeta_{A^{\prime}}$ with $\alpha^{A^{\prime}}$ constant. An unprimed spinor (i.e. an element of $S$ ) is an object of the form $\zeta^{A} h_{A}$ with $\zeta^{A}$ constant. The 2 -form $e_{A^{\prime} B^{\prime}}$ on $S^{\prime}$ is found by substituting (4.4) into (4.2) and using the definition (2.20): this gives

$$
e^{\prime}(\alpha, \beta)=-J^{-1} \varepsilon_{A^{\prime} B^{\prime}} \alpha^{A^{\prime}} \beta^{B^{\prime}} .
$$

The 2-form $e_{A B}$ on $S$ is computed according to the definition (2.19) and turns out to be

$$
e(\zeta, \mu)=\frac{1}{2} \varepsilon_{A B} \zeta^{A} \mu^{B} .
$$

The metric $g_{a b}$ is now obtained by putting together (4.5), (4.6) and (4.7):

$$
\begin{aligned}
& g_{a b} d x^{a} d x^{b}=\frac{1}{2}\left(-J^{-1}\right) \Omega \\
& \quad=J^{-2}[(1+Y \tilde{Y}) d X d \tilde{X}+(1+X \tilde{X}) d Y d \tilde{Y}-X \tilde{Y} d Y d \tilde{X}-Y \tilde{X} d X d \tilde{Y}] .
\end{aligned}
$$

This is indeed the Fubini-Study metric on $P_{2}$, on the 'real slice' $\tilde{X}=\bar{X}, \tilde{Y}=\bar{Y}$. For example, it is identical to that in [11], (10), where $\zeta^{1}=X, \zeta^{2}=Y$ and (their $\Lambda)=6$. The metric (4.8) has scalar curvature $R=-\frac{1}{24}$, as expected from (4.3). (The curvature tensor used in [11] is of opposite sign to ours.)

\section{Appendix}

We have to prove that the forms $\tau$ and $\rho$, defined by (2.5) and (2.6), satisfy

$$
\tau \wedge d \tau=2 \Lambda \rho
$$

First it is necessary to compute $d \tau$. To do so, we follow an unpublished argument due to Penrose. Let $W$ be a 2-surface in $\mathscr{T}$. Thus $W$ corresponds to a 2 parameter family of $\alpha$-surfaces in $M$. We make the assumption that the $\alpha$-surfaces in this family do not intersect one another. In other words, the two-parameter family of 2-surfaces make up a foliation of $M$ : exactly one $\alpha$-surface in the family $W$ passes through each point of $M$. If we can define $d \tau$ on the generic 2-surface $W$ in $\mathscr{T}$ satisfying this assumption, then this will suffice to determine $d \tau$ on $\mathscr{T}$.

The 'foliation' assumption means that the spinor fields $\pi_{A^{\prime}}$ on each $\alpha$-surface in the family $W$ combine into a spinor field $\pi_{A^{\prime}}$ on the whole space $M$, satisfying

$$
\pi^{A^{\prime}} \nabla_{A A^{\prime}} \pi_{B^{\prime}}=0 .
$$


Let $\sigma$ be the projection map from $M$ to $W$; in other words,

$$
\sigma(x)=\mathbf{Z} \in W \Leftrightarrow x \in Z .
$$

Let $\zeta$ be the 1 -form on $M$ defined by $\zeta=\left(\pi_{B^{\prime}} \nabla_{a} \pi^{B^{\prime}}\right) d x^{a}$. In fact, $\zeta$ is the pull-back of $\tau$ to $M$ : if $v$ is a vector field on $M$, then

$$
\begin{aligned}
\left(\sigma^{*} \tau\right)(v) & =\tau\left(\sigma_{*} v\right) & & \left(\text { definition of } \sigma^{*}\right) \\
& =\pi_{B^{\prime}} \mathrm{v}^{a} \nabla_{a} \pi^{B^{\prime}} & & \text { (definition of } \tau) \\
& =\zeta(v) . & & (\text { definition of } \zeta)
\end{aligned}
$$

So to compute $d \tau$, it suffices to compute $d \zeta$ (remember that the pull-back operator $\sigma^{*}$ commutes with the exterior derivative operator: $\left.\sigma^{*}(d \tau)=d\left(\sigma^{*} \tau\right)=d \zeta\right)$.

To compute $d \zeta$ we may proceed as follows. Let $v_{1}$ and $v_{2}$ be two vector fields on $M$ which are constant over the $\alpha$-surfaces in the family $W$, i.e. $\pi^{A^{\prime}} \nabla^{A A^{\prime}} \mathrm{v}_{1}{ }^{b}=0=$ $\pi^{A^{\prime}} \nabla_{A A^{\prime}} v_{2}^{b}$. Then

$$
\begin{aligned}
d \zeta\left(v_{1}, v_{2}\right) & =\frac{1}{2} v_{1}{ }^{c} \nabla_{c}\left(\pi_{B^{\prime}} v_{2}{ }^{a} \nabla_{a} \pi^{B^{\prime}}\right)-(1 \leftrightarrow 2) \\
& =\beta_{1 A^{\prime}} \beta_{2}{ }^{A^{\prime}}+\Lambda \alpha_{1 A}{ }^{{ }^{A}},
\end{aligned}
$$

where $\beta_{1 A^{\prime}}=\mathrm{v}_{1}{ }^{b} \nabla_{b} \pi_{A^{\prime}}, \alpha_{1}{ }^{A}=i v_{1}{ }^{A A^{\prime}} \pi_{A^{\prime}}$ etc. and where the Ricci identities $(1.7,1.8)$ have been used. Thus we conclude that if $Q_{1} \leftrightarrow\left(\alpha_{1}{ }^{A}, \beta_{1 A^{\prime}}\right)$ and $Q_{2} \leftrightarrow\left(\alpha_{2}{ }^{A}, \beta_{2 A^{\prime}}\right)$ are two vectors in $\mathscr{T}$, then

$$
d \tau\left(Q_{1}, Q_{2}\right)=\beta_{1 A^{\prime}} \beta_{2}^{A^{\prime}}+\Lambda \alpha_{1 A^{\alpha}}{ }^{A} .
$$

As a check, one may verify, using (2.3) and (2.4), that the right hand side of (A2) is indeed constant over $\alpha$-surfaces; and so $d \tau$ is well-defined.

Finally, (2.5) and (A1.3) give

$$
\begin{aligned}
\tau \wedge d \tau\left(Q_{1}, Q_{2}, Q_{3}\right) & =\pi_{A^{\prime}} \beta_{1}^{A^{\prime}}\left(\beta_{2 B^{\prime}} \beta_{3}{ }^{B^{\prime}}+\Lambda \alpha_{2 B} \alpha_{3}{ }^{B}\right) \text { skewed over } 1,2,3 \\
& =2 \Lambda \rho,
\end{aligned}
$$

as is seen from eq. (2.6). The term involving three $\beta$ 's vanishes because the $\beta$ 's are elements of a two-dimensional vector space, so skewing over more than two of them produces zero.

Acknowledgment. The author is indebted to R. Penrose and M. F. Atiyah for many useful conversations. Most of the work in this paper was carried out while the author was a Junior Research Fellow at Merton College, Oxford.

\section{References}

1. Penrose, R. : Gen. Rel. Grav. 7, 31-52 (1976)

2. Newman, E. T. : Gen. Rel. Grav. 7, 107-111 (1976)

3. Plebanski, J. F. : J. Math. Phys. 16, 2395-2402 (1975)

4. Atiyah, M. F., Hitchin, N. J., Singer, I. M. : Proc. R. Soc. London A362, 425-461 (1978)

5. Hawking, S. W.: The path-integral approach to quantum gravity. In: General relativity (eds. S. W. Hawking, W. Israel), pp. 746-789. Cambridge: Cambridge University Press 1979

6. Ward, R. S. : Proc. R. Soc. London A363, 289-295 (1978)

7. Hitchin, N. J. : Proc. Cambridge Philos. Soc. 85, 465-476 (1979)

8. Hitchin, N. J. : Proc. R. Soc. London A370, 173-191 (1980) 
9. Atiyah, M. F. : Green's functions for self-dual 4-manifolds. Adv. Math. To appear

10. Eguchi, T., Freund, P.G.O. : Phys. Rev. Lett. 37, 1251-1254 (1976)

11. Gibbons, G. W., Pope, C. B. : Commun. Math. Phys. 61, 239-248 (1978)

12. Penrose, R.: The structure of space-time. In: Battelle rencontres (eds. C. M. De Witt, J. A. Wheeler), pp. 121-235. New York: Benjamin 1968

13. Penrose, R., MacCallum, M.A.H. : Phys. Reports 6C, 241-315(1972)

14. Curtis, W. D., Lerner, D. E., Miller, F. R. : Gen. Rel. Grav. 10, 557-565 (1979)

15. Kodaira, K. : Am. J. Math. 85, 79-94 (1963)

16. Hansen, R. O., Newman, E. T., Penrose, R., Tod, K. P. : Proc. R. Soc. London A363, 445-468 (1978)

17. Ward, R, S. : Phys. Letters A61, 81-82 (1977)

Communicated by R. Geroch

Received December 13, 1979 
